\title{
INTERSECTIONS OF SYMBOLIC POWERS OF PRIME IDEALS
}

\author{
SEAN SATHER-WAGSTAFF
}

\begin{abstract}
Let $(R, \mathfrak{m})$ be a local ring with prime ideals $\mathfrak{p}$ and $\mathfrak{q}$ such that $\sqrt{\mathfrak{p}+\mathfrak{q}}=\mathfrak{m}$. If $R$ is regular and contains a field, and $\operatorname{dim}(R / \mathfrak{p})+\operatorname{dim}(R / \mathfrak{q})=\operatorname{dim}(R)$, we prove that $\mathfrak{p}^{(m)} \cap \mathfrak{q}^{(n)} \subseteq \mathfrak{m}^{m+n}$ for all positive integers $m$ and $n$. This is proved using a generalization of Serre's Intersection Theorem which we apply to a hypersurface $R / f R$. The generalization gives conditions that guarantee that Serre's bound on the intersection dimension $\operatorname{dim}(R / \mathfrak{p})+\operatorname{dim}(R / \mathfrak{q}) \leq \operatorname{dim}(R)$ holds when $R$ is nonregular.
\end{abstract}

\section{Introduction}

Let $k$ be an algebraically closed field of characteristic 0 and $\mathbb{A}_{k}^{d}$ the $d$ dimensional affine space over $k$. That is, $\mathbb{A}_{k}^{d}=\operatorname{Spec}(S)$ where $S$ is the polynomial algebra $k\left[X_{1}, \ldots, X_{d}\right]$. Let $Y$ and $Z$ be closed subvarieties of $\mathbb{A}_{k}^{d}$ that intersect at a finite number of points, and assume without loss of generality that the intersection contains the origin. Let $f$ be a nonzero regular function on $\mathbb{A}_{k}^{n}$ that vanishes on $Y \cup Z$, and let $m$ and $n$ denote the orders of vanishing of $f$ along $Y$ and $Z$, respectively. That is, all the partial derivatives of $f$ of order at most $(m-1)$ vanish identically on $Y$ and at least one $m$ th order partial does not, and similarly for $n$. It is reasonable to ask, in this case, what the order of vanishing of $f$ is at the origin. Of course, if

\footnotetext{
${ }^{0} 2000$ Mathematics Subject Classification 13H05, 13H15, 13C15, 13D22
} 
$r=\max \{m, n\}$ then $f$ vanishes to order at least $r$, and the following easy example shows that, without additional hypotheses, this bound is sharp.

Example 1.1. Let $d=3, Y=V\left(X_{1}, X_{2}\right)$ and $Z=V\left(X_{2}, X_{3}\right)$. Then $Y$ and $Z$ intersect precisely at the origin, and the function $X_{2}$ vanishes exactly to order 1 at every point of $Y$ and at every point of $Z$. In particular, $X_{2}$ vanishes exactly to order 1 at the origin.

One may feel somewhat cheated by this example since $X_{2}$ is an "essential" defining polynomial for both subvarieties. The existence of this polynomial is objectionable for another related reason. In our example, we are considering two lines in 3-space, and in general, two such varieties will not intersect. In fact, our lines intersect exactly because they are coplanar, and more specifically because they live in the plane defined by the vanishing of the "offending" polynomial $X_{2}$. If we consider two lines in general position, that is if we "wiggle" the lines slightly, then they will generally be pulled away from one another; the question of vanishing of functions at an intersection point is then meaningless. This suggests that we should demand that the objects under consideration be well-behaved under "wiggling." In order to guarantee this, we recall a classical theorem from algebraic geometry which states that, under our initial hypotheses, $\operatorname{dim}(Y)+\operatorname{dim}(Z) \leq d$. We shall refer to this as the Classical Intersection Theorem. In addition, when $\operatorname{dim}(Y)+\operatorname{dim}(Z)=d$, the varieties will intersect generically at a finite nonempty set of points. In Example 1.1 this inequality is strict, and we can directly attribute this to the smooth hypersurface $V\left(X_{2}\right)$ containing $Y$ and $Z$. What can be said about orders of vanishing if we assume that the subvarieties satisfy the additional hypothesis $\operatorname{dim}(Y)+\operatorname{dim}(Z)=d$ ? To motivate our answer, we consider the 
following example.

Example 1.2. Assume that $d$ is at least 2 and fix an integer $i$ between 1 and $d-1$. Let $\mathfrak{p}=\left(X_{1}, \ldots, X_{i}\right) S, \mathfrak{q}=\left(X_{i+1}, \ldots, X_{d}\right) S, Y=V(\mathfrak{p})$ and $Z=V(\mathfrak{q})$. Then $Y$ and $Z$ intersect at exactly the origin and $\operatorname{dim}(Y)+\operatorname{dim}(Z)=d$. Zariski's Main Lemma on Holomorphic Functions [25] implies that a nonzero regular function $f$ vanishes to order $m$ at every point of $Y$ if and only if $f \in \mathfrak{p}^{(m)}$, where $\mathfrak{p}^{(m)}=R \cap \mathfrak{p}^{m} R_{\mathfrak{p}}$ is the $m$ th symbolic power of $\mathfrak{p}$. In this case, the ordinary and symbolic powers of $\mathfrak{p}$ agree, so $f$ vanishes to order $m$ at every point of $Y$ if and only if $f \in \mathfrak{p}^{m}$. Similarly, $f$ vanishes to order $n$ at every point of $Z$ if and only if $f \in \mathfrak{q}^{(n)}=\mathfrak{q}^{n}$. It is straightforward to show that, in this case

$$
\mathfrak{p}^{(m)} \cap \mathfrak{q}^{(n)}=\mathfrak{p}^{m} \cap \mathfrak{q}^{n}=\mathfrak{p}^{m} \mathfrak{q}^{n} \subseteq \mathfrak{m}^{m+n} .
$$

Therefore $f$ vanishes at the origin to order at least $m+n$. That is, the sum of the orders of vanishing along $Y$ and $Z$ gives a lower bound for the order of vanishing at the origin. One can easily construct examples to show that this bound is sharp.

This example leads us to ask the following question.

Question 1.3. Let $k$ be an algebraically closed field of characteristic 0 , and $Y$ and $Z$ closed subvarieties of $\mathbb{A}_{k}^{d}$ that intersect at finitely many points, including the origin, and such that $\operatorname{dim}(Y)+\operatorname{dim}(Z)=d$. If $f$ is a nonzero regular function on $\mathbb{A}_{k}^{n}$ vanishing along $Y$ and $Z$ to orders $m$ and $n$, respectively, does $f$ vanish to order at least $m+n$ at the origin?

As a corollary to one of the main results of this paper, we answer this question in the affirmative. See Corollary 2.4 below. 
The discussion preceding in Example 1.2 suggests a purely algebraic formulation of Question 1.3. We state the local version as part of Conjecture 1.6 below. Before we do so, we present a somewhat more algebraic motivation for this question. We include it, not only because the methods we use to answer Question 1.3 are purely algebraic in nature, but also because it indicates how we originally came to consider these ideas.

Let $(R, \mathfrak{m})$ be a local, Noetherian ring with prime ideals $\mathfrak{p}$ and $\mathfrak{q}$ such that $\sqrt{\mathfrak{p}+\mathfrak{q}}=\mathfrak{m}$. Serre [23] generalized the Classical Intersection Theorem by proving that, if $R$ is regular, then

$$
\operatorname{dim}(R / \mathfrak{p})+\operatorname{dim}(R / \mathfrak{q}) \leq \operatorname{dim}(R)
$$

We shall refer to this result as Serre's Intersection Theorem. When $R / \mathfrak{p}$ has finite projective dimension (true automatically if $R$ is regular) Serre defined the intersection multiplicity of $R / \mathfrak{p}$ and $R / \mathfrak{q}$ as

$$
\chi(R / \mathfrak{p}, R / \mathfrak{q})=\sum_{i=0}^{\operatorname{dim}(R)}(-1)^{i} \ell\left(\operatorname{Tor}_{i}^{R}(R / \mathfrak{p}, R / \mathfrak{q})\right)
$$

where $\ell$ is the length function. When $R$ is regular and unramified, he proved that $\chi(R / \mathfrak{p}, R / \mathfrak{q}) \geq 0$ with strict inequality holding if and only if $\operatorname{dim}(R / \mathfrak{p})+$ $\operatorname{dim}(R / \mathfrak{q})=\operatorname{dim}(R)$. He conjectured that the same holds true if the ring is ramified.

Independently, Roberts [18] and Gillet-Soulé [5] proved the vanishing part of the conjecture in the ramified case: if $\operatorname{dim}(R / \mathfrak{p})+\operatorname{dim}(R / \mathfrak{q})<\operatorname{dim}(R)$ then $\chi(R / \mathfrak{p}, R / \mathfrak{q})=0$. Gabber [1], 8, 19] proved nonnegativity: $\chi(R / \mathfrak{p}, R / \mathfrak{q}) \geq 0$. The Positivity Conjecture is the converse to the vanishing result and is still open in the ramified case. 
By applying Gabber's methods to the Positivity Conjecture, Kurano and Roberts [12] proved the following.

Theorem 1.4. Let $(R, \mathfrak{m})$ be a regular local ring with prime ideals $\mathfrak{p}$ and $\mathfrak{q}$ such that $\sqrt{\mathfrak{p}+\mathfrak{q}}=\mathfrak{m}$ and $\operatorname{dim}(R / \mathfrak{p})+\operatorname{dim}(R / \mathfrak{q})=\operatorname{dim}(R)$. If $R$ is ramified or contains a field and $\chi(R / \mathfrak{p}, R / \mathfrak{q})>0$, then $\mathfrak{p}^{(m)} \cap \mathfrak{q} \subseteq \mathfrak{m}^{m+1}$ for all positive integers $m$, where $\mathfrak{p}^{(m)}$ is the $m$ th symbolic power of $\mathfrak{p}$.

Because Kurano and Roberts expect the Positivity Conjecture to be true, this result motivated them to make the following conjecture.

Conjecture 1.5. Let $(R, \mathfrak{m})$ be a regular local ring with prime ideals $\mathfrak{p}$ and $\mathfrak{q}$ such that $\sqrt{\mathfrak{p}+\mathfrak{q}}=\mathfrak{m}$ and $\operatorname{dim}(R / \mathfrak{p})+\operatorname{dim}(R / \mathfrak{q})=\operatorname{dim}(R)$. Then $\mathfrak{p}^{(m)} \cap \mathfrak{q} \subseteq$ $\mathfrak{m}^{m+1}$ for all positive integers $m$.

There is a significant amount of interest in the properties of symbolic powers of prime ideals in regular local rings. In addition to the paper of Kurano-Roberts, the interested reader should refer to [4, 9, [10, 11].

Theorem 1.4 shows that Conjecture 1.5 is true for regular rings containing an arbitrary field since positivity holds for these rings. In their paper, Kurano and Roberts verify the conjecture, with no reference to positivity, when $R$ contains a field of characteristic 0 . They prove this by first demonstrating that, if $\mathfrak{p} \subseteq \mathfrak{m}^{2}$ then $\mathfrak{p}^{(m)} \subseteq \mathfrak{m}^{m+1}$ for all $m \geq 1$; they then reduce the general case to the case where $\mathfrak{p} \subseteq \mathfrak{m}^{2}$. Curiously, the proof makes little reference to the second prime $\mathfrak{q}$. The current author [21] has verified the conjecture, again with no reference to positivity, when $R$ contains a field of arbitrary characteristic. This is accomplished by first proving a generalization of Serre's Intersection Theorem and applying it to a hypersurface $R / f R$. 
We discuss the interaction between such generalizations and Conjecture 1.5 (and its generalizations) in greater depth below. We note that Conjecture 1.5 is still open in mixed-characteristic.

Theorem 1.4 is immediately applicable to Question 1.3, as follows. Let $Y$ and $Z$ be closed subvarieties of $\mathbb{A}_{k}^{d}$ that intersect in a finite number of points, including the origin, and such that $\operatorname{dim}(Y)+\operatorname{dim}(Z)=d$. Let $f$ be a nonzero function on $\mathbb{A}_{k}^{n}$ that vanishes to order $m$ and $n$ along $Y$ and $Z$, respectively. Without loss of generality, assume that $m \geq n$. Let $\mathfrak{p}$ and $\mathfrak{q}$ be the prime ideals of $S$ defining $Y$ and $Z$, respectively, and let $\mathfrak{m}=\left(X_{1}, \ldots, X_{d}\right) S$. The function $f$ is an element of $\mathfrak{p}^{(m)}$ by Zariski's Main Lemma, and it is an element of $\mathfrak{q}$ by assumption. It follows from Theorem 1.4 that

$$
f \in S \cap \mathfrak{m}^{m+1} S_{\mathfrak{m}}=\mathfrak{m}^{(m+1)}=\mathfrak{m}^{m+1} .
$$

In other words, $f$ vanishes to order at least $m+1$ at the origin. Although this does not answer Question 1.3, it at least demonstrates that the assumption $\operatorname{dim}(Y)+\operatorname{dim}(Z)=d$ implies greater order of vanishing than demonstrated in Example 1.1.

In considering Conjecture 1.5 with Question 1.3 in mind, one might be tempted to ask why the conjecture is not more symmetric. (This is, of course, an easy question to ask in light of the discussion above. However, one might also be led to this question by observing the symmetry of the intersection multiplicity $\chi$ and its relevance to the conjecture via Theorem 1.4.) The main reason is that the evidence that led to the formulation of the conjecture was Theorem 1.4, and at the time there was no similar result suggesting stronger results for $\mathfrak{p}^{(m)} \cap \mathfrak{q}^{(n)}$. In addition, one notices that the proof of Theorem 1.4 is not symmetric, as one of the first steps is to take a regular alteration 
of $\operatorname{Spec}(R / \mathfrak{p})$ ] and pass to a closely related projective scheme. Therefore, Kurano and Roberts did not necessarily expect a symmetric result.

As the earlier discussion indicates, though, we do consider a more symmetric intersection in the current paper. More specifically, under the hypotheses of Conjecture 1.5, we ask whether the containment $\mathfrak{p}^{(m)} \cap \mathfrak{q}^{(n)} \subseteq$ $\mathfrak{m}^{m+n}$ holds. In one of our main results we answer this question in the affirmative for rings containing an arbitrary field. More specifically, we have the following.

Theorem 2.3 Let $(R, \mathfrak{m})$ be a regular local ring containing a field and $\mathfrak{p}$ and $\mathfrak{q}$ prime ideals of $R$ such that $\sqrt{\mathfrak{p}+\mathfrak{q}}=\mathfrak{m}$ and $\operatorname{dim}(R / \mathfrak{p})+\operatorname{dim}(R / \mathfrak{q})=\operatorname{dim}(R)$. Then $\mathfrak{p}^{(m)} \cap \mathfrak{q}^{(n)} \subseteq \mathfrak{m}^{m+n}$ for all $m, n \geq 1$.

As an immediate consequence of this result, we are able to answer Question 1.3 in the affirmative.

Theorem 2.3 is established by proving the following generalization of Serre's Intersection Theorem for nonregular rings and applying it to the hypersurface $R / f R$ where $f$ is an element of $\mathfrak{p}^{(m)} \cap \mathfrak{q}^{(n)}$.

Theorem 2.2 Let $(A, \mathfrak{n})$ be a quasi-unmixed local ring and assume that one

\footnotetext{
${ }^{1} \mathrm{~A}$ regular alteration of $\operatorname{Spec}(R / \mathfrak{p})$ is similar to a resolution of singularities: it is a projective morphism $\phi: X \rightarrow \operatorname{Spec}(R / \mathfrak{p})$ where $X$ is a regular scheme. Regular alterations are weaker than resolutions of singularities because they do not necessarily induce isomorphisms on the fields of rational functions, only finite extensions. However, unlike resolutions of singularities, they are known to exist for rings essentially of finite type over a field (of arbitrary characteristic) or a complete discrete valuation ring, by the work of de Jong [3]. The existence of regular alterations is key to Gabber's proof of the nonnegativity conjecture.
} 
of the following conditions holds.

1. A red $_{\text {contains a field, or }}$

2. A is a ring of mixed-characteristic such that the residual characteristic $p$ is part of a reductive system of parameters of $A$.

Let $P$ and $Q$ be prime ideals in $A$ such that both $A / P$ and $A / Q$ are analytically unramified, $\sqrt{P+Q}=\mathfrak{n}$, and $e(A)<e\left(A_{P}\right)+e\left(A_{Q}\right)$. Then $\operatorname{dim}(A / P)+\operatorname{dim}(A / Q) \leq \operatorname{dim}(A)$.

When we say that $A$ is quasi-unmixed, we mean that every irreducible component of the completion $A^{*}$ has the same dimension, that is, that $A^{*}$ is equidimensional. By a theorem of Ratliff [17], this is equivalent to $A$ being equidimensional and universally catenary. The ring $A_{\text {red }}$ is the reduced ring $A / \operatorname{nil}(A)$, and $e(A)$ is the Hilbert-Samuel multiplicity of $A$. A reductive system of parameters for $A$ is a system of parameters that generates a reduction of $\mathfrak{n}$. (See Section 2 for complete definitions and relevant properties.) Briefly, the connection between Theorems 2.2 and 2.3 is the fact that, if $0 \neq f \in \mathfrak{p}$, then $e\left(R_{\mathfrak{p}} / f R_{\mathfrak{p}}\right)=m$ if and only if $f \in \mathfrak{p}^{(m)} \backslash \mathfrak{p}^{(m+1)}$.

This leads us to consider two conjectural generalizations of Serre's Intersection Theorem. Each statement generalizes a question about symbolic powers of prime ideals in regular local rings. We list the conjectures here with the corresponding conjectures for symbolic powers.

Conjecture 1.6. Let $(A, \mathfrak{n})$ be a quasi-unmixed local ring with prime ideals $P$ and $Q$ such that $\sqrt{P+Q}=\mathfrak{n}$. Also, let $(R, \mathfrak{m})$ be a regular local ring with prime ideals $\mathfrak{p}$ and $\mathfrak{q}$ such that $\sqrt{\mathfrak{p}+\mathfrak{q}}=\mathfrak{m}$ and $\operatorname{dim}(R / \mathfrak{p})+\operatorname{dim}(R / \mathfrak{q})=$ $\operatorname{dim}(R)$. Then 
(SP-1) $\mathfrak{p}^{(m)} \cap \mathfrak{q} \subseteq \mathfrak{m}^{m+1}$ for all $m \geq 1$.

(SP-2) $\mathfrak{p}^{(m)} \cap \mathfrak{q}^{(n)} \subseteq \mathfrak{m}^{m+n}$ for all $m, n \geq 1$

(ID-1) If $e(A)=e\left(A_{P}\right)$ and $A / P$ is analytically unramified, then

$$
\operatorname{dim}(A / P)+\operatorname{dim}(A / Q) \leq \operatorname{dim}(A) .
$$

(ID-2) If $e(A)<e\left(A_{P}\right)+e\left(A_{Q}\right)$ and both $A / P$ and $A / Q$ are analytically unramified, then

$$
\operatorname{dim}(A / P)+\operatorname{dim}(A / Q) \leq \operatorname{dim}(A) .
$$

We immediately observe that (ID-1) and (ID-2) are conjectural generalization of Serre's Intersection Theorem. This is due to the fact that, if $A$ is regular then $e(A)=e\left(A_{P}\right)=e\left(A_{Q}\right)=1$. Conjecture (SP-1) and (ID-1) have been verified for rings containing an arbitrary field and for a number of special cases in 12, 21, 22], and Theorems 2.3 and 2.2 of the present paper imply that (SP-2) and (ID-2) are true for rings containing fields.

We note that ours are not the first efforts made to generalize Serre's Intersection Theorem to nonregular rings. Of course, there is the landmark paper of Peskine and Szpiro [16], as well as the more recent work of Simon [24].

Below, we discuss the reasons for the technical assumptions in Conjecture 1.6. Before we do so, we discuss the relations between the individual conjectures. Obviously, (SP-2) implies (SP-1). If (ID-2) holds for all complete hypersurfaces, then (SP-2) holds for all regular local rings. To see this, pass to the completion $R^{*}$ of $R$ and apply (ID-2) as in the proof of Theorem 2.3. Similarly, if (ID-1) is true for all complete hypersurfaces, then 
(SP-1) is true for all regular local rings. Clearly (ID-2) implies (ID-1) when $A / Q$ is analytically unramified, because $e\left(A_{Q}\right)>0$ and $e\left(A_{P}\right)=e(A)$ imply that $e(A)<e\left(A_{P}\right)+e\left(A_{Q}\right)$. It is straightforward to reduce (ID-1) to the case of a complete domain - we perform this reduction explicitly for (ID-2) in the proof of Theorem 2.2 and it follows that (ID-2) implies (ID-1). We summarize the implications is the following diagram.

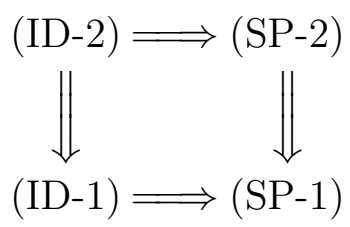

By Theorem 1.4, Serre's Positivity Conjecture implies (SP-1) for ramified rings. At this time, we do not know whether positivity implies (SP-1) for unramified rings or any of the other conjectures in any case of mixed characteristic. In addition we do not know whether any of these conjectures imply Serre's Positivity Conjecture.

Here we discuss the technical assumptions of Conjecture 1.6. It is easy to find examples to show why $R$ must be regular, $\sqrt{\mathfrak{p}+\mathfrak{q}}$ must equal $\mathfrak{m}$, and $\operatorname{dim}(R / \mathfrak{p})+\operatorname{dim}(R / \mathfrak{q})$ must equal $\operatorname{dim}(R)$. Similarly, one sees that $\sqrt{P+Q}$ must equal $\mathfrak{n}$. The ring $A$ must be equidimensional in (ID-1) and (ID-2), by considering $A=k \llbracket X, Y, Z \rrbracket /(X Y, X Z)$ and letting $P$ and $Q$ be the minimal primes of $A$. In [22] Example 6.5, we give an example showing that $A$ should be at least catenary. We may or may not need the full strength of quasi-unmixed, that is, equidimensional and universally catenary. In our arguments, where we pass to the completion, we need the completion to be equidimensional. If $A$ is excellent and equidimensional, then it is automatic, so we do not consider this assumption too restrictive. 
When we wish to use $e\left(A_{P}\right)$, we assume that $A / P$ is analytically unramified. By this, we mean that the completion $(A / P)^{*}=A^{*} / P A^{*}$ is reduced, or in other words, the ideal $P A^{*}$ is an intersection of prime ideals of $A^{*}$. The purpose of this assumption is to guarantee that the multiplicity is wellbehaved under localization. More specifically, we require that the multiplicity not increase after localizing. Our guarantee is from Nagata [14] (40.1).

Theorem 1.7. Let $P$ be a prime ideal of a local ring $A$. If $A / P$ is analytically unramified and if ht $(P)+\operatorname{dim}(A / P)=\operatorname{dim}(A)$, then $e\left(A_{P}\right) \leq e(A)$.

Regarding the analytically unramified assumption, Nagata 14 (Appendix A2) wrote the following. "It is not yet known to the writer's knowledge whether or not (40.1) is true without assuming that $P$ is analytically unramified." (The statement " $P$ is analytically unramified" means " $A / P$ is analytically unramified.") If it is shown that this condition can be omitted from the statement of Theorem 1.7, then the corresponding conditions should probably be omitted from Conjectures (ID-1) and (ID-2). For now, however, we leave them intact, especially because our arguments (in which we pass to the completion) depend on the assumptions. As with the quasi-unmixedness assumption, if $A$ is excellent then $A / P$ is guaranteed to be analytically unramified, so this is not an unbearable restriction.

Acknowledgments: I am grateful to S. Dutta, P. Griffith, G. Leuschke and A. Singh for their helpful comments and suggestions. 


\section{The Main Results}

Before we verify the conjectures for rings containing fields, we give some definitions and background results. An excellent reference for multiplicitytheoretic results is Herrmann, Ikeda and Orbanz [6].

If $(A, \mathfrak{n})$ is a local ring and $M$ is a nonzero, finitely generated $A$-module of dimension $d$ and $I$ is an ideal of $A$ such that $M / I M$ has finite length, then the Hilbert function $P(n)=\ell_{A}\left(M / I^{n+1} M\right)$ is a polynomial of degree $d$ for $n \gg 0$. The Hilbert-Samuel multiplicity is the positive integer $e_{A}(I, M)$ such that $P(n)=\frac{1}{d !} e_{A}(I, M) n^{d}+$ (lower degree terms). When there is no danger of confusion, we shall write $e(I, M)$. If $I=\mathfrak{n}$, we write $e_{A}(M)$ or $e(M)$.

Regarding the Hilbert-Samuel multiplicity, we shall use the following facts freely and possibly without reference.

1. (Associativity Formula) Let $A$ be a local ring with finitely generated module $M$. Then

$$
e_{A}(M)=\sum_{P} \ell\left(M_{P}\right) e(A / P)
$$

where the sum is taken over all prime ideals $P$ in the support of $M$ such that $\operatorname{dim}(A / P)=\operatorname{dim}(M)$. In particular, this sum is finite.

2. Let $(R, \mathfrak{m})$ be a local ring contained in a ring $A$ such that the extension $R \hookrightarrow A$ is module-finite. Then $A$ is a semilocal ring, say with maximal ideals $\mathfrak{n}_{1}, \ldots, \mathfrak{n}_{n}$, such that $\operatorname{dim}(A)=\operatorname{dim}(R)$ and each $\mathfrak{n}_{i} \cap R=\mathfrak{m}$. Then

$$
e_{R}(\mathfrak{m}, A)=\sum_{i}\left[A / \mathfrak{n}_{i}: R / \mathfrak{m}\right] e_{A_{\mathfrak{n}_{i}}}\left(\mathfrak{m} A_{\mathfrak{n}_{i}}, A_{\mathfrak{n}_{i}}\right)
$$


where the sum is taken over all indices $i$ such that ht $\left(\mathfrak{n}_{i}\right)=\operatorname{dim}(A)$.

3. Let $R$ be a Noetherian ring contained in a ring $A$ such that the extension $R \hookrightarrow A$ is module-finite, and let $\mathfrak{s}$ be a prime ideal of $R$. Let $\left\{S_{1}, \ldots, S_{j}\right\}$ be the set of prime ideals of $A$ such that $S_{i} \cap R=\mathfrak{s}$ and ht $\left(S_{i}\right)=\operatorname{ht}(\mathfrak{s})$. Then

$$
e_{R_{\mathfrak{s}}}\left(\mathfrak{s} R_{\mathfrak{s}}, A_{\mathfrak{s}}\right)=\sum_{i=1}^{j}\left[\kappa\left(S_{i}\right): \kappa(\mathfrak{s})\right] e_{A_{S_{i}}}\left(\mathfrak{s} A_{S_{i}}, A_{S_{i}}\right)
$$

where $\kappa(\mathfrak{s})$ is the residue field of $R_{\mathfrak{s}}$ and similarly for $\kappa\left(S_{i}\right)$.

4. Let $A \rightarrow A^{\prime}$ be a flat, local homomorphism of local rings $(A, \mathfrak{n})$ and $\left(A^{\prime}, \mathfrak{n}^{\prime}\right)$ such that $\mathfrak{n} A^{\prime}=\mathfrak{n}^{\prime}$. Then $e\left(A^{\prime}\right)=e(A)$.

5. Let $A$ be a local domain and $I$ an ideal of $A$ such that $A / I$ has finite length. If $M$ is an $A$-module of positive rank $r$, then

$$
e_{A}(I, M)=e_{A}(I, A) \cdot r .
$$

Property 1 is Bruns and Herzog [2] Corollary 4.7.8. Property 2 is Nagata [14] (23.1). Property 3 is simply (2) applied to the extension $R_{\mathfrak{s}} \rightarrow A_{\mathfrak{s}}$. Property 4 is Herzog [7] Lemma 2.3. And property 5 is Matsumura [13] Theorem 14.8.

The following is the most important tool used to prove out main results.

Lemma 2.1. Let $(A, \mathfrak{n})$ be an equidimensional, catenary local ring containing a catenary local domain $(R, \mathfrak{m})$ such that the extension $R \hookrightarrow A$ is modulefinite. Let $P$ and $Q$ be prime ideals of $A$ such that $\sqrt{P+Q}=\mathfrak{n}$ and both $A / P$ and $A / Q$ are analytically unramified, and let $\mathfrak{p}=P \cap R$ and $\mathfrak{q}=Q \cap R$. 
Assume that the quotient $R / \mathfrak{s}$ is analytically unramified for every prime ideal $\mathfrak{s} \supset \mathfrak{p}+\mathfrak{q}$. If $e_{R}(\mathfrak{m}, A)<e\left(A_{P}\right)+e\left(A_{Q}\right)$, then $\sqrt{\mathfrak{p}+\mathfrak{q}}=\mathfrak{m}$.

If $R$ is an excellent local domain and $A$ is equidimensional, then $A$ is also excellent. In this case, $A$ is automatically catenary and the quotients $A / P, A / Q$ and $R / \mathfrak{s}$ are guaranteed to be analytically unramified. In our applications, both $A$ and $R$ will in fact be complete, so the reader is free to assume either of these stronger conditions.

Proof. Let $\mathfrak{s}$ be a prime ideal of $R$ such that $\mathfrak{p}+\mathfrak{q} \subseteq \mathfrak{s}$. To show that $\sqrt{\mathfrak{p}+\mathfrak{q}}=\mathfrak{m}$, it suffices to show that $\mathfrak{s}=\mathfrak{m}$. By the going-up property for integral extensions, fix prime ideals $S_{1}$ and $S_{2}$ of $A$ such that $S_{1} \supseteq P, S_{2} \supseteq Q$ and $S_{1} \cap R=S_{2} \cap R=\mathfrak{s}$. To show that $\mathfrak{s}=\mathfrak{m}$, it suffices to show that $S_{1}=S_{2}$ because then $S_{1} \supseteq P+Q$ so that $S_{1}=\mathfrak{n}$ and $\mathfrak{s}=S_{1} \cap R=\mathfrak{n} \cap R=\mathfrak{m}$.

Suppose that $S_{1} \neq S_{2}$. We first observe that, for every prime $S$ of $A$ such that $S \cap R=\mathfrak{s}$, we have ht $(S)=$ ht $(\mathfrak{s})$. This is due to the fact that, by integrality $\operatorname{dim}(R / \mathfrak{s})=\operatorname{dim}(A / S)$, and the catenary and equidimensional assumptions imply that ht $(S)=\operatorname{dim}(A)-\operatorname{dim}(A / S)$ and similarly for $\mathfrak{s}$. In particular, ht $\left(S_{i}\right)=$ ht $(\mathfrak{s})$ for $i=1,2$. Thus

$$
\begin{array}{rlr}
e\left(A_{P}\right)+e\left(A_{Q}\right) & >e_{R}(\mathfrak{m}, A)=\operatorname{rank}_{R}(A) e(R) & (\text { by }(\mathbb{q})) \\
& \geq \operatorname{rank}_{R_{\mathfrak{s}}}\left(A_{\mathfrak{s}}\right) e\left(R_{\mathfrak{s}}\right) & \text { (by Theorem 1.7) } \\
& =e_{R_{\mathfrak{s}}}\left(\mathfrak{s} R_{\mathfrak{s}}, A_{\mathfrak{s}}\right) & \text { (by (国)) } \\
& =\sum_{S \cap R=\mathfrak{s}}[\kappa(S): \kappa(\mathfrak{s})] e_{A_{S}}\left(\mathfrak{s} A_{S}, A_{S}\right) &
\end{array}
$$


(where the sum is taken over all primes $S$ of $A$ contracting to $\mathfrak{s}$ in $R$, by (3))

$$
\begin{aligned}
& \geq e\left(\mathfrak{s} A_{S_{1}}, A_{S_{1}}\right)+e\left(\mathfrak{s} A_{S_{2}}, A_{S_{2}}\right) \\
& \geq e\left(S_{1} A_{S_{1}}, A_{S_{1}}\right)+e\left(S_{2} A_{S_{2}}, A_{S_{2}}\right) \\
& =e\left(A_{S_{1}}\right)+e\left(A_{S_{2}}\right) .
\end{aligned}
$$

By [14] (36.8) the rings $A_{S_{1}} / P A_{S_{1}}$ and $A_{S_{2}} / Q A_{S_{2}}$ are analytically unramified. Therefore, Theorem 1.7 implies that

$$
e\left(A_{P}\right)+e\left(A_{Q}\right)>e\left(A_{S_{1}}\right)+e\left(A_{S_{2}}\right) \geq e\left(A_{P}\right)+e\left(A_{Q}\right) .
$$

This clearly gives a contradiction.

Given two ideals $I \supseteq J$ in a ring $A$, we say that $J$ is a reduction of $I$ if there is a positive integer $n$ such that $I^{n+1}=J I^{n}$. If $(A, \mathfrak{n})$ is local and $I$ is a reduction of $\mathfrak{n}$, then $e(I, A)=e(A)$. If $A$ is local with infinite residue field, then there is a system of parameters of $A$ that generates a reduction of $\mathfrak{n}$ (c.f., Northcott and Rees [15]). We shall call such a system of parameters a reductive system of parameters of $A$. If $A$ has infinite residue field then Herrmann, Ikeda and Orbanz [6] Proposition 10.17 says that an element $x$ is part of a reductive system of parameters of $A$ if and only if the initial form of $x$ in the associated graded ring $\operatorname{gr}_{\mathfrak{n}}(A)$ is part of a homogeneous system of parameters of $\operatorname{gr}_{\mathfrak{n}}(A)$.

The following theorem shows that (ID-2) holds for rings containing a field and for a certain class of mixed-characteristic rings.

Theorem 2.2. Let $(A, \mathfrak{n})$ be a quasi-unmixed local ring and assume that one of the following conditions holds. 


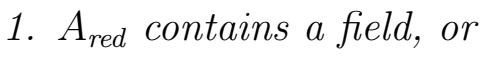

2. A is a ring of mixed-characteristic such that the residual characteristic $p$ is part of a reductive system of parameters of $A$.

Let $P$ and $Q$ be prime ideals in $A$ such that both $A / P$ and $A / Q$ are analytically unramified, $\sqrt{P+Q}=\mathfrak{n}$, and $e(A)<e\left(A_{P}\right)+e\left(A_{Q}\right)$. Then $\operatorname{dim}(A / P)+\operatorname{dim}(A / Q) \leq \operatorname{dim}(A)$.

In the proof of this result we demonstrate that (ID-2) need only be verified for complete domains with infinite residue fields.

Proof. Step 1. Pass to the $\operatorname{ring} A(X)=A[X]_{\mathfrak{n} A[X]}$ to assume that the residue field of $A$ is infinite. Let $\mathfrak{n}(X)=\mathfrak{n} A(X), P(X)=P A(X)$ and $Q(X)=$ $Q A(X)$. Then $A(X)$ is a local ring with maximal ideal $\mathfrak{n}(X)$. If $A_{\text {red }}$ contains a field, then the same is true of $A(X)_{\text {red }}$. If the sequence $p, x_{2}, \ldots, x_{d}$ is a reductive system of parameters of $A$, then the same sequence is a reductive system of parameters of $A(X)$ since for some $t$,

$$
\mathfrak{n}(X)^{t+1}=\mathfrak{n}^{t+1} A(X)=\left(p, x_{2}, \ldots, x_{d}\right) \mathfrak{n}^{t} A(X)
$$

Multiplicities are preserved by property 5 at the beginning of this section. That is, $e(A(X))=e(A), e\left(A(X)_{P(X)}\right)=e\left(A_{P}\right)$ and $e\left(A(X)_{Q(X)}\right)=e\left(A_{Q}\right)$. Both rings $A(X) / P(X)$ and $A(X) / Q(X)$ are analytically unramified by Nagata [14 (36.8). Thus, if the result holds in $A(X)$, then

$$
\begin{aligned}
\operatorname{dim}(A / P)+\operatorname{dim}(A / Q) & =\operatorname{dim}(A(X) / P(X))+\operatorname{dim}(A(X) / Q(X)) \\
& \leq \operatorname{dim}(A(X))=\operatorname{dim}(A)
\end{aligned}
$$

as desired. 
Step 2. Pass to the completion $\left(A^{*}, \mathfrak{n}^{*}\right)$ to assume that $A$ is complete and equidimensional with infinite residue field. Let $P^{*}$ be a prime ideal of $A^{*}$ that is minimal over $P A^{*}$ such that ht $\left(P^{*}\right)=\operatorname{ht}(P)$, and similarly for $Q^{*}$. Since $A / P$ is analytically unramified, $P A_{P^{*}}^{*}=P^{*} A_{P^{*}}^{*}$. The fact that the extension $A_{P} \rightarrow A_{P^{*}}^{*}$ is flat therefore implies that $e\left(A_{P^{*}}^{*}\right)=e\left(A_{P}\right)$ by property 5 at the beginning of this section. Similarly, $e\left(A_{Q^{*}}^{*}\right)=e\left(A_{Q}\right)$. If $A_{\text {red }}$ contains a field, then the same is true of $\left(A^{*}\right)_{\text {red }}$. If the sequence $p, x_{2}, \ldots, x_{d}$ is a reductive system of parameters of $A$, then the same sequence is a reductive system of parameters of $A^{*}$, as in the previous step. If the result holds for $A^{*}$, then it holds for $A$, as in the previous step.

Step 3. Pass to the quotient $A / I$ for a suitably chosen minimal prime $I$, to assume that $A$ is a complete domain with infinite residue field, and that either $A$ itself contains a field or $A$ satisfies hypothesis 2 in the statement of the theorem. To make this reduction, it suffices to verify that there is a minimal prime $I$ of $A$ contained in $P \cap Q$ such that $e(A / I)<e\left(A_{P} / I A_{P}\right)+e\left(A_{Q} / I A_{Q}\right)$.

First, we show how this gives the desired reduction. Let $A^{\prime}=A / I$ with prime ideals $\mathfrak{n}^{\prime}=\mathfrak{n} A^{\prime}, P^{\prime}=P A^{\prime}$ and $Q^{\prime}=\mathfrak{q} A^{\prime}$. If $A_{\text {red }}$ contains a field, then $A^{\prime}$ contains a field since $A^{\prime}$ is a quotient of $A_{\text {red. }}$. If the sequence $p, x_{2}, \ldots, x_{d}$ is a reductive system of parameters of $A$, then the same sequence is a reductive system of parameters of $A^{\prime}$, as in Step 2. Since $A$ is equidimensional, $\operatorname{dim}\left(A^{\prime}\right)=\operatorname{dim}(A)$. And since $I \subseteq P \cap Q$, it follows that $\operatorname{dim}\left(A^{\prime} / P^{\prime}\right)=\operatorname{dim}(A / P)$ and $\operatorname{dim}\left(A^{\prime} / Q^{\prime}\right)=\operatorname{dim}(A / Q)$. Therefore, as in Step 1, we may pass to $A^{\prime}$.

Now we prove that such a minimal prime $I$ exists. Let $\left\{I_{1}, \ldots, I_{g}\right\}=$ $\min (A)$. Suppose that $e\left(A / I_{j}\right) \geq e\left(A_{P} / I_{j} A_{P}\right)+e\left(A_{Q} / I_{j} A_{Q}\right)$ for every $j$ such 
that $I_{j} \subseteq P \cap Q$. (This supposition includes the hypothetical possibility that no $I_{j}$ is contained in $P \cap Q$.) By Theorem 1.7, $e\left(A_{P} / I_{j} A_{P}\right) \leq e\left(A / I_{j}\right)$ for every $I_{j}$ contained in $P$, and similarly for $I_{j}$ contained in $Q$. The Associativity Formula then implies that

$$
\begin{aligned}
& e\left(A_{P}\right)+e\left(A_{Q}\right)=\sum_{I_{j} \subseteq P} e\left(A_{P} / I_{j} A_{P}\right) \ell\left(A_{I_{j}}\right)+\sum_{I_{j} \subseteq Q} e\left(A_{Q} / I_{j} A_{Q}\right) \ell\left(A_{I_{j}}\right)
\end{aligned}
$$

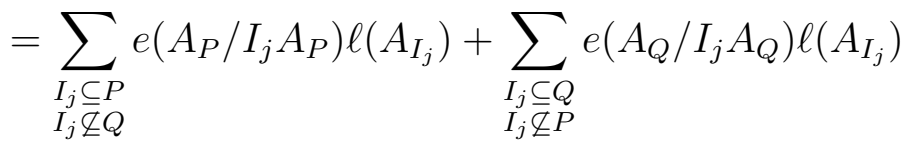

$$
\begin{aligned}
& +\sum_{I_{j} \subseteq P \cap Q}\left[e\left(A_{P} / I_{j} A_{P}\right)+e\left(A_{Q} / I_{j} A_{Q}\right)\right] \ell\left(A_{I_{j}}\right) \\
& \leq \sum_{\substack{I_{j} \subseteq P \\
I_{j} \subseteq Q}} e\left(A / I_{j}\right) \ell\left(A_{I_{j}}\right)+\sum_{\substack{I_{j} \subseteq Q \\
I_{j}}} e\left(A / I_{j}\right) \ell\left(A_{I_{j}}\right) \\
& +\sum_{I_{j} \subseteq P \cap Q} e\left(A / I_{j}\right) \ell\left(A_{I_{j}}\right) \\
& \leq \sum_{j} e\left(A / I_{j}\right) \ell\left(A_{I_{j}}\right) \\
& =e(A) \text {. }
\end{aligned}
$$

This clearly contradicts the assumption that $e(A)<e\left(A_{P}\right)+e\left(A_{Q}\right)$. Thus, there is a minimal prime $I$ of $A$ such that $I \subseteq P \cap Q$ and $e(A / I)<$ $e\left(A_{P} / I A_{P}\right)+e\left(A_{Q} / I A_{Q}\right)$, as claimed.

Step 4. We prove the result assuming that $A$ is a complete domain with infinite residue field. If $A$ contains a field, let $x_{1}, \ldots, x_{d}$ be a reductive system of parameters of $A, k$ a coefficient field of $A, R=k \llbracket x_{1}, \ldots, x_{d} \rrbracket$, and $\mathfrak{m}=$ $\left(x_{1}, \ldots, x_{d}\right) R$. If $A$ has mixed characteristic, then by assumption there is a reductive system of parameters $p, x_{2}, \ldots, x_{d}$ of $A$. By the Cohen Structure Theorem there is a complete discrete valuation ring $(V, p V)$ contained in $A$ 
such that the induced map on residue fields is an isomorphism. In this case, let $R=V \llbracket x_{2}, \ldots, x_{d} \rrbracket$ and $\mathfrak{m}=\left(p, x_{2}, \ldots, x_{d}\right) R$. Note that, in either case $R$ is a regular local ring contained in $A$ such that the extension $R \rightarrow A$ is module-finite. The maximal ideal of $R$ is $\mathfrak{m}$ and the extension ideal $\mathfrak{m} A$ is a reduction of $\mathfrak{n}$. Furthermore, the induced maps on residue fields is an isomorphism. Therefore,

$$
\begin{aligned}
e_{R}(\mathfrak{m}, A) & =[A / \mathfrak{n}: R / \mathfrak{m}] e_{A}(\mathfrak{m} A, A) \\
& =e_{A}(\mathfrak{n}, A)=e(A)<e\left(A_{P}\right)+e\left(A_{Q}\right)
\end{aligned}
$$

If $\mathfrak{p}=P \cap R$ and $\mathfrak{q}=Q \cap R$, then Lemma 2.1 implies that $\sqrt{\mathfrak{p}+\mathfrak{q}}=\mathfrak{m}$, and Serre's Intersection Theorem implies that

$$
\operatorname{dim}(A / P)+\operatorname{dim}(A / Q)=\operatorname{dim}(R / \mathfrak{p})+\operatorname{dim}(R / \mathfrak{q}) \leq \operatorname{dim}(R)=\operatorname{dim}(A)
$$

This completes the proof.

The following theorem is one of our main results and our main application of Theorem 2.2.

Theorem 2.3. Let $(R, \mathfrak{m})$ be a regular local ring containing a field and $\mathfrak{p}$ and $\mathfrak{q}$ prime ideals of $R$ such that $\sqrt{\mathfrak{p}+\mathfrak{q}}=\mathfrak{m}$ and $\operatorname{dim}(R / \mathfrak{p})+\operatorname{dim}(R / \mathfrak{q})=$ $\operatorname{dim}(R)$. Then $\mathfrak{p}^{(m)} \cap \mathfrak{q}^{(n)} \subseteq \mathfrak{m}^{m+n}$ for all $m, n \geq 1$.

Proof. Fix a nonzero element $f \in \mathfrak{p}^{(m)} \cap \mathfrak{q}^{(n)}$ and without loss of generality, assume that $f \notin \mathfrak{p}^{(m+1)}$ and $f \notin \mathfrak{q}^{(n+1)}$. Let $A=R / f R$ with maximal ideal $\mathfrak{n}=\mathfrak{m} A$ and prime ideals $P=\mathfrak{p} A$ and $Q=\mathfrak{q} A$. If $f \notin \mathfrak{m}^{m+n}$, then $e(A)<m+n=e\left(A_{P}\right)+e\left(A_{Q}\right)$. Theorem 2.2 implies that

$$
\operatorname{dim}(R / \mathfrak{p})+\operatorname{dim}(R / \mathfrak{q})=\operatorname{dim}(A / P)+\operatorname{dim}(A / Q) \leq \operatorname{dim}(A)=\operatorname{dim}(R)-1
$$


which gives a contradiction.

It is now straightforward to answer Question 1.3 in the affirmative, and we do so explicitly in the following corollary.

Corollary 2.4. Let $k$ be an algebraically closed field of characteristic 0, and $Y$ and $Z$ closed subvarieties of $\mathbb{A}_{k}^{d}$ that intersect at finitely many points, including the origin, and such that $\operatorname{dim}(Y)+\operatorname{dim}(Z)=d$. If $f$ is a nonzero regular function on $\mathbb{A}_{k}^{d}$ vanishing along $Y$ and $Z$ to orders $m$ and $n$, respectively, then $f$ vanishes to order at least $m+n$ at the origin.

Proof. Let $\mathfrak{p}$ and $\mathfrak{q}$ be the prime ideals of $R=k\left[X_{1}, \ldots, X_{d}\right]$ such that $Y=V(\mathfrak{p})$ and $Z=V(\mathfrak{q})$, and let $\mathfrak{m}=\left(X_{1}, \ldots, X_{d}\right) R$. The aforementioned result of Zariski implies that $f$ is an element of $\mathfrak{p}^{(m)} \cap \mathfrak{q}^{(n)}$. Passing the the regular local ring $R_{\mathfrak{m}}$ we have

$$
f \in \mathfrak{p}^{(m)} R_{\mathfrak{m}} \cap \mathfrak{q}^{(n)} R_{\mathfrak{m}}=\left(\mathfrak{p} R_{\mathfrak{m}}\right)^{(m)} \cap\left(\mathfrak{q} R_{\mathfrak{m}}\right)^{(n)} \subseteq\left(\mathfrak{m} R_{\mathfrak{m}}\right)^{m+n}=\mathfrak{m}^{m+n} R_{\mathfrak{m}}
$$

which implies that $f$ is in the ideal $\mathfrak{m}^{(m+n)}=\mathfrak{m}^{m+n}$, as desired.

In the following corollary we state an immediate generalization of Theorem 2.3. It is easy to see that the corresponding result for mixed characteristic will hold once we verify (SP-2) for such rings. Recall that a not necessarily local) Noetherian ring is said to be regular if the localization at every maximal ideal is a regular local ring.

Corollary 2.5. Let $R$ be a regular ring containing a field with prime ideals $\mathfrak{p}_{1}, \ldots, \mathfrak{p}_{t}$. Let $\mathfrak{m}$ be a minimal prime ideal of $\mathfrak{p}_{1}+\cdots+\mathfrak{p}_{t}$ and assume that $\operatorname{ht}(\mathfrak{m})=\sum_{i} \operatorname{ht}\left(\mathfrak{p}_{i}\right)$. Then,

$$
\mathfrak{p}_{1}^{\left(n_{1}\right)} \cap \cdots \cap \mathfrak{p}_{t}^{\left(n_{t}\right)} \subseteq \mathfrak{m}^{\left(\sum_{i} n_{i}\right)}
$$


for all $n_{1}, \ldots, n_{t} \geq 1$.

One version of Serre's Intersection Theorem states that, since $\mathfrak{m}$ is minimal over $\sum_{i} \mathfrak{p}_{i}$, ht $(\mathfrak{m}) \leq \sum_{i}$ ht $\left(\mathfrak{p}_{i}\right)$. Thus, the assumption that ht $(\mathfrak{m})=$ $\sum_{i}$ ht $\left(\mathfrak{p}_{i}\right)$ simply assures that ht $(\mathfrak{m})$ is as large as possible.

Proof. By passing to the localization $R_{\mathfrak{m}}$, we assume without loss of generality that $R$ is regular local with maximal ideal $\mathfrak{m}$. We prove the result in this case by induction on $t$. When $t=1$, the result is trivial. When $t=2$, this is exactly Theorem 2.3. Assume now that $t \geq 3$. Fix a prime ideal $\mathfrak{s}$ minimal over $\sum_{i \geq 2} \mathfrak{p}_{i}$. Then by Serre's Intersection Theorem, ht $(\mathfrak{s}) \leq \sum_{i \geq 2}$ ht $\left(\mathfrak{p}_{1}\right)$. Since $\mathfrak{p}_{1}+\mathfrak{s}$ is $\mathfrak{m}$-primary, $\sum_{i \geq 1}$ ht $\left(\mathfrak{p}_{1}\right)=\mathrm{ht}(\mathfrak{m}) \leq \mathrm{ht}\left(\mathfrak{p}_{1}\right)+\mathrm{ht}(\mathfrak{s})$ and it follows that ht $(\mathfrak{s})=\sum_{i \geq 2}$ ht $\left(\mathfrak{p}_{1}\right)$. Therefore, by induction

$$
\mathfrak{p}_{1}^{\left(n_{1}\right)} \cap \cdots \cap \mathfrak{p}_{t}^{\left(n_{t}\right)} \subseteq \mathfrak{p}_{1}^{\left(n_{1}\right)} \cap \mathfrak{s}^{\left(\sum_{i \geq 2} n_{i}\right)} \subseteq \mathfrak{m}^{\left(\sum_{i \geq 1} n_{i}\right)}
$$

as desired.

In the following corollary, similar to Theorem 2.3, we give a partial verification of (SP-2) in the unramified case of mixed-characteristic.

Corollary 2.6. Let $(R, \mathfrak{m}, k)$ be an unramified regular local ring of mixedcharacteristic $p$. Let $\mathfrak{p}$ and $\mathfrak{q}$ be prime ideals of $R$ such that $\sqrt{\mathfrak{p}+\mathfrak{q}}=\mathfrak{m}$ and $\operatorname{dim}(R / \mathfrak{p})+\operatorname{dim}(R / q)=d$ and fix a nonzero element $f \in \mathfrak{p}^{(m)} \cap \mathfrak{q}^{(n)}$. In the associated graded ring $\operatorname{gr}_{\mathfrak{m}}(R)$, let the initial forms of $f$ and $p$ be denoted by $F$ and $P$, respectively. If $F$ is not divisible by $P$, then $f \in \mathfrak{m}^{m+n}$.

Proof. By passing to the completion $R^{*}$ of the $\operatorname{ring} R(X)=R[X]_{\mathfrak{m} R[X]}$, we may assume without loss of generality that $R$ is complete with infinite residue field. The only nontrivial observation is that, if $\operatorname{gr}_{\mathfrak{m}}(R)=$ 
$k\left[P, X_{1}, \ldots, X_{d}\right]$, then the associated graded rings of both $R(X)$ and $R^{*}$ are $k(X)\left[P, X_{1}, \ldots, X_{d}\right]$. If $P$ does not divide $F$ in $k\left[P, X_{1}, \ldots, X_{d}\right]$, then the same is true in $k(X)\left[P, X_{1}, \ldots, X_{d}\right]$.

The remainder of the proof is similar to that of Theorem 2.3. The only difference is that, in the application of Theorem 2.2 we must verify that $p$ is part of a reductive system of parameters of $A=R / f R$. Note that the associated graded ring of $A$ is $\operatorname{gr}_{\mathfrak{n}}(A)=k\left[P, X_{2}, \ldots, X_{d}\right] /(F)$. The fact that $k$ is infinite and $P$ does not divide $F$ implies that $P$ is part of a homogeneous system of parameters of degree 1 for $\operatorname{gr}_{\mathfrak{n}}(A)$. As noted in the discussion preceding Theorem 2.2, this implies that $p$ is part of a reductive system of parameters of $A$. Thus, Theorem 2.2 applies.

\section{Special Cases}

We quickly demonstrate three special cases where (SP-2) holds. First, we prove the case when one of the ideals is generated by part of a regular system of parameters of $R$.

Proposition 3.1. Let $(R, \mathfrak{m})$ be a regular local ring with prime ideals $\mathfrak{p}$ and $\mathfrak{q}$ such that $\sqrt{\mathfrak{p}+\mathfrak{q}}=\mathfrak{m}$ and $\operatorname{dim}(R / \mathfrak{p})+\operatorname{dim}(R / \mathfrak{q})=\operatorname{dim}(R)$. If $R / \mathfrak{p}$ is regular, then $\mathfrak{p}^{(m)} \cap \mathfrak{q}^{(n)} \subseteq \mathfrak{p}^{(m)} \mathfrak{m}^{n} \subseteq \mathfrak{m}^{m+n}$ for all $m, n \geq 1$.

Proof. Since $R / \mathfrak{p}$ is regular, $\mathfrak{p}$ is generated by elements $x_{1}, \ldots, x_{h}$ that form part of a regular system of parameters of $R$. Complete this to a regular system of parameters $\mathbf{x}=x_{1}, \ldots, x_{h}, \ldots, x_{d}$ for $R$. The fact that $\mathbf{x}$ is a regular sequence and $R$ is Cohen-Macaulay implies that $\mathfrak{p}^{(m)}=\mathfrak{p}^{m}$ for all $m \geq 1$. 
It suffices to show that $\mathfrak{p}^{(m)} \cap \mathfrak{q}^{(n)} \subseteq \mathfrak{m}^{m+n}$ for all $m, n \geq 1$. To see that this is sufficient, observe that, because $\mathfrak{p}$ is generated by part of the regular sequence that generates $\mathfrak{m}$,

$$
\mathfrak{p}^{(m)} \mathfrak{m}^{n-1} \cap \mathfrak{m}^{m+n}=\mathfrak{p}^{(m)} \mathfrak{m}^{n}
$$

even when $n=1$. The desired sufficiency now follows by induction on $n$.

By passing to the ring $R(X)=R[X]_{\mathfrak{m} R[X]}$ as in the proof of Theorem 2.2, we may assume without loss of generality that the residue field of $R$ is infinite. We prove that $\mathfrak{p}^{(m)} \cap \mathfrak{q}^{(n)} \subseteq \mathfrak{m}^{m+n}$ by induction on $h$. If $h=0$ or $h=1$ then $\mathfrak{p}$ is principal. Since $\mathfrak{q}$ is prime, it is straightforward to show that, in either of these cases, $\mathfrak{p}^{(m)} \cap \mathfrak{q}^{(n)}=\mathfrak{p}^{m} \mathfrak{q}^{(n)} \subseteq \mathfrak{m}^{m+n}$.

Now assume that $h \geq 2$. Since $R / \mathfrak{m}$ is infinite, we choose an infinite sequence $a_{1}, a_{2}, \ldots \in R$ such that the residues $\overline{a_{i}}$ of the $a_{i}$ modulo $\mathfrak{m}$ are all distinct. Let $y_{i}=x_{1}+a_{i} x_{2} \in \mathfrak{p}$ and for each $i$ choose a prime ideal $\mathfrak{q}_{i}$ containing $\mathfrak{q}$ and $y_{i}$ such that ht $\left(\mathfrak{q}_{i}\right)=$ ht $(\mathfrak{q})+1$. The quotient $R_{i}=R / y_{i} R$ with prime ideals $\mathfrak{p} R_{i}$ and $\mathfrak{q}_{i} R_{i}$ satisfies the induction hypothesis. Suppose that $f$ is a nonzero element of $\mathfrak{p}^{(m)} \cap \mathfrak{q}^{(n)} \subseteq \mathfrak{p}^{(m)} \cap \mathfrak{q}_{i}^{(n)}$. Then the residue of $f$ in $R_{i}$ is in $\left(\mathfrak{p} R_{i}\right)^{(m)} \cap\left(\mathfrak{q}_{i} R_{i}\right)^{(n)} \subseteq\left(\mathfrak{m} R_{i}\right)^{m+n}$ so that $f \in y_{i} R+\mathfrak{m}^{m+n}$ for every $i$. The associated graded ring of $R$ is the polynomial $\operatorname{ring} \operatorname{gr}_{\mathfrak{m}}(r)=$ $k\left[X_{1}, \ldots, X_{d}\right]$ where $X_{i}$ is the initial form of $x_{i}$. If $f \notin \mathfrak{m}^{m+n}$, then the fact that $f$ is in the ideal $y_{i} R+\mathfrak{m}^{m+n}$ for every $i$ implies that the initial form $F$ of $f$ in $\operatorname{gr}_{\mathfrak{m}}(R)$ is divisible by the initial form of $y_{i}$. That is, $F$ is divisible by $Y_{i}=X_{1}+\overline{a_{i}} X_{2}$ for every $i$. Since the $\overline{a_{i}}$ are all distinct in $R / \mathfrak{m}$, the $Y_{i}$ all generate distinct prime ideals in $k\left[X_{1}, \ldots, X_{d}\right]$. Thus, $F$ has an infinite number of distinct, irreducible factors, contradicting the fact that $k\left[X_{1}, \ldots, X_{d}\right]$ is a unique factorization domain. 
In the following proposition, we verify (SP-2) when $\mathfrak{p}$ and $\mathfrak{q}$ are both generated by regular sequences. We say that an ideal $I$ in a ring $A$ is unmixed if $\operatorname{dim}(A / P)=\operatorname{dim}(A / I)$ for every $P \in \operatorname{Ass}(A / I)$. In this case, we define the $n$th symbolic power of $I$ to be

$$
I^{(n)}=\cap_{P}\left(I^{n} A_{P} \cap A\right)
$$

where the intersection is taken over all associated primes $P$ of $A / I$. If $I$ is prime, this agrees with the standard definition of symbolic powers. If $A$ is Cohen-Macaulay and $I$ is generated by a regular sequence, then $I$ is unmixed, and for all $n \geq 1, I^{(n)}=I^{n}$ and $A / I^{n}$ is Cohen-Macaulay.

Proposition 3.2. Let $(R, \mathfrak{m})$ be a regular local ring with ideals $I$ and $J$ such that $\sqrt{I+J}=\mathfrak{m}$ and $\operatorname{dim}(R / I)+\operatorname{dim}(R / J)=\operatorname{dim}(R)$. If both $I$ and $J$ are generated by regular sequences, then $I^{(m)} \cap J^{(n)}=I^{m} J^{n} \subseteq \mathfrak{m}^{m+n}$ for all $m, n \geq 1$.

Proof. By assumption, $R / I^{(m)}$ and $R / J^{(n)}$ are Cohen-Macaulay modules such that $\operatorname{dim}\left(R / I^{(m)}\right)+\operatorname{dim}\left(R / J^{(n)}\right)=\operatorname{dim}(R)$. By Serre [23] Corollary to Theorem 4 in Chapter V, we know that $\operatorname{Tor}_{i}\left(R / I^{(m)}, R / J^{(n)}\right)=0$. By Rotman 20 Corollary 11.27 (iii),

$$
\left(I^{(m)} \cap J^{(n)}\right) / I^{m} J^{n}=\left(I^{m} \cap J^{n}\right) / I^{m} J^{n}=\operatorname{Tor}_{1}\left(R / I^{m}, R / J^{n}\right)=0
$$

and it follows that $\left(I^{(m)} \cap J^{(n)}\right)=I^{m} J^{n} \subseteq \mathfrak{m}^{m+n}$.

In the following proposition, we verify (SP-2) for graded primes in a polynomial algebra. 
Proposition 3.3. Let $p$ be a prime number, $(T, p T)$ a discrete valuation ring, $R=T\left[X_{1}, \ldots, X_{d}\right]$ with the standard grading, and $\mathfrak{m}=\left(p, X_{1}, \ldots, X_{d}\right) R$. Let $\mathfrak{p}$ and $\mathfrak{q}$ be homogeneous prime ideals of $R$ such that $\sqrt{\mathfrak{p}+\mathfrak{q}}=\mathfrak{m}$ and $\operatorname{dim}(R / \mathfrak{p})+\operatorname{dim}(R / \mathfrak{q})=d+1$. Then $\mathfrak{p}^{(m)} \cap \mathfrak{q}^{(n)} \subseteq \mathfrak{m}^{m+n}$ for all $m, n \geq 1$.

Proof. By passing to the ring $R(Y)=T(Y)\left[X_{1}, \ldots, X_{d}\right]$ as in our previous arguments, we may assume without loss of generality that $T$ has infinite residue field. The ordinary power $\mathfrak{p}^{m}$ is a homogeneous ideal, so that its $\mathfrak{p}$-primary component $\mathfrak{p}^{(m)}$ is also homogeneous. Similarly, $\mathfrak{q}^{(n)}$ is also homogeneous. Therefore, to check the desired inclusion, we need only check homogeneous elements. Let $f$ be a homogeneous element of $\mathfrak{p}^{(m)} \cap \mathfrak{q}^{(n)}$. Write $f$ as a product $f=p^{s} f_{1} \cdots f_{t}$ where each $f_{i}$ is a nonconstant, irreducible, homogeneous polynomial of degree $d_{i}$.

Because $\mathfrak{p}$ and $\mathfrak{q}$ are homogeneous and $\sqrt{\mathfrak{p}+\mathfrak{q}}=\mathfrak{m}$, it is straightforward to show that $p$ is an element of either $\mathfrak{p}$ or $\mathfrak{q}$. We assume without loss of generality that $p \in \mathfrak{p}$. Then $p^{s} \in \mathfrak{p}^{(s)} \backslash \mathfrak{p}^{(s+1)}$. By Serre's Intersection Theorem applied to the ring $R / p R$, our assumptions imply that $p$ can not be in both $\mathfrak{p}$ and $\mathfrak{q}$.

Let $e_{i}=\max \left\{e: f_{i} \in \mathfrak{p}^{(e)}\right\}$ and $e_{i}^{\prime}=\max \left\{e: f_{i} \in \mathfrak{q}^{(e)}\right\}$ for $i=1, \ldots, t$. Then, $s+\sum_{i} e_{i} \geq m, \sum_{i} e_{i}^{\prime} \geq n$, and each $f_{i} \in \mathfrak{p}^{\left(e_{i}\right)} \cap \mathfrak{q}^{\left(e_{i}^{\prime}\right)}$. If each $f_{i} \in \mathfrak{m}^{e_{i}+e_{i}^{\prime}}$ (this is automatic if either $e_{i}=0$ or $e_{i}^{\prime}=0$ ) then

$$
f=p^{s} f_{1} \cdots f_{t} \in \mathfrak{m}^{s+\sum_{i}\left(e_{i}+e_{i}^{\prime}\right)} \subseteq \mathfrak{m}^{m+n}
$$

as desired. Thus, we may assume without loss of generality that $f$ is irreducible and not divisible by $p$. In particular, $f$ has positive degree and some monomial term of $f$ has unit coefficient. This implies that, in the associated 
graded ring of the localization $R_{\mathfrak{m}}$, the initial form of $f$ has a monomial term with unit coefficient. Therefore, by Corollary 2.6 $f \in \mathfrak{m}^{(m+n)}=\mathfrak{m}^{m+n}$.

\section{References}

[1] P. Berthelot, 'Altérations de variétés algébriques (d'après A. J. de Jong)', Astérisque (1997), Exp. No. 815, 5, 273-311, Séminaire Bourbaki, Vol. 1995/96.

[2] W. Bruns and J. Herzog, Cohen-Macaulay rings, (Cambridge University Press, Cambridge, 1993).

[3] A. J. de Jong, 'Smoothness, semi-stability and alterations', Inst. Hautes Études Sci. Publ. Math. (1996), 51-93.

[4] D. Eisenbud and B. Mazur, 'Evolutions, symbolic squares, and Fitting ideals', J. Reine Angew. Math. 488 (1997), 189-201.

[5] H. Gillet and C. Soulé, ' $K$-théorie et nullité des multiplicités d'intersection', C. R. Acad. Sci. Paris Sér. I Math. 300 (1985), 71-74.

[6] M. Herrmann, S. Ikeda, and U. Orbanz, Equimultiplicity and blowing up, An algebraic study, With an appendix by B. Moonen (Springer-Verlag, Berlin, 1988).

[7] B. Herzog, 'On the Macaulayfication of local rings', J. Algebra 67 (1980), 305-317. 
[8] M. Hochster, 'Nonnegativity of intersection multiplicities in ramified regular local rings following Gabber/De Jong/Berthelot', unpublished notes, available at

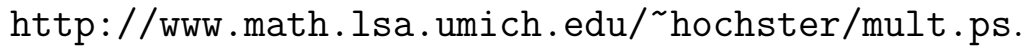

[9] M. Hochster and C. Huneke, 'Comparison of symbolic and ordinary powers of ideals', preprint, available at http://www.math. Isa.umich.edu/ hochster/sym.ps.

[10] S. Huckaba and C. Huneke, 'Powers of ideals having small analytic deviation', Amer. J. Math. 114 (1992), 367-403.

[11] C. Huneke and J. Ribbe, 'Symbolic squares in regular local rings', Math. Z. 229 (1998), 31-44.

[12] K. Kurano and P. Roberts, 'The positivity of intersection multiplicities and symbolic powers of prime ideals', Compositio Math. 122 (2000), $165-182$.

[13] H. Matsumura, Commutative ring theory (Cambridge University Press, Cambridge, 1986).

[14] M. Nagata, Local rings (Interscience Publishers a division of John Wiley \& Sons New York-London, 1962).

[15] D. G. Northcott and D. Rees, 'Reductions of ideals in local rings', Proc. Cambridge Philos. Soc. 50 (1954), 145-158.

[16] C. Peskine and L. Szpiro, 'Syzygies et multiplicités', C. R. Acad. Sci. Paris Sér. A 278 (1974), 1421-1424. 
[17] L. Ratliff, Jr., 'On quasi-unmixed local domains, the altitude formula, and the chain condition for prime ideals. II', Amer. J. Math. 92 (1970), 99-144.

[18] P. Roberts, 'The vanishing of intersection multiplicities of perfect complexes', Bull. Amer. Math. Soc. (N.S.) 13 (1985), 127-130.

[19] P. Roberts, 'Recent developments on Serre's multiplicity conjectures: Gabber's proof of the nonnegativity conjecture', Enseign. Math. (2) 44 (1998), 305-324.

[20] J. Rotman, An introduction to homological algebra (Academic Press Inc. [Harcourt Brace Jovanovich Publishers], New York, 1979).

[21] S. Sather-Wagstaff, 'A dimension inequality for Cohen-Macaulay rings', Trans. Amer. Math. Soc. (to appear)

[22] S. Sather-Wagstaff, 'Muliplicities and a dimension inequality for unmixed modules', J. Algebra. 238 (2001), 372-388.

[23] J-P. Serre, Algèbre locale. Multiplicités, (Springer-Verlag, Berlin, 1965).

[24] A-M. Simon, 'Intersection dimension and canonical modules', Rings, Hopf algebras, and Brauer groups (Antwerp/Brussels, 1996), (eds S. Caenepeel and A. Verschoren), Lecture Notes in Pure and Applied Mathematics, 197 (Dekker, New York, 1998), pp. 287-297.

[25] O. Zariski, 'A fundamental lemma from the theory of holomorphic functions on an algebraic variety', Ann. Mat. Pura Appl. (4) 29 (1949), $187-198$ 
Department of Mathematics

University of Illinois

273 Altgeld Hall,

1409 West Green Street

Urbana IL, 61801

ssather@math.uiuc.edu 